\title{
Does Social Risk Amplification Theory Explain the Resistance to COVID-19 Geo-Localization Applications?
}

\author{
Mohamed Nabil Mzoughi ${ }^{1}$, Karim Garrouch ${ }^{2}$ \\ ${ }^{1}$ Department of Marketing, College of Business, Dar Al Uloom University, Riyadh, Saudi Arabia \\ ${ }^{2}$ Department of Business Administration, College of Administrative and Financial Sciences, \\ Saudi Electronic University, Riyadh. Saudi Arabia
}

\begin{abstract}
KSA have launched a mobile application which recognizes if there is a confirmed COVID-19 subject in proximity. Its adoption can be explained by technology acceptance models, but the pandemic context involves also the use of a theory dealing with the health risk. This study adapts the Social Amplification of Risk Framework to verify a model explaining three behaviors: information seeking, preventive behavior and resistance to COVID-19 mobile application Tawakkalna. A survey has been distributed to a sample of 312 individuals living in Saudi Arabia.

Findings show that media coverage and perceived risk have an impact on negative affective reactions which influence behavior.
\end{abstract}

Keywords - Mobile application, COVID-19, Resistance, prevention behavior, perceived risk.

\section{Introduction}

COVID-19 is a pandemic whose solution, at a governmental level, is based on social distancing measures aiming at slowing down or stopping its expansion [1].

DOI: 10.18421/TEM103-26

https://doi.org/10.18421/TEM103-26

Corresponding author: Mohamed Nabil Mzoughi, Dar Al Uloom University, Riyadh, Saudi Arabia.

Email: m.nabil@dau.edu.sa

Received: 07 April 2021.

Revised: 15 July 2021.

Accepted: 19 July 2021.

Published: 27 August 2021.

(cc) BY-NC-ND (C) 2021 Mohamed Nabil Mzoughi \& Karim Garrouch; published by UIKTEN. This work is licensed under the Creative Commons AttributionNonCommercial-NoDerivs 4.0 License.

The article is published with Open Access at www.temjournal.com
To keep public awareness and overcome this pandemic, many governments launched mobile applications which use and share information about infected subjects in proximity [2].

KSA authorities have launched the mobile application Tawakalna which recognizes if there is a confirmed Covid-19 subject in proximity. The adoption of this application can be explained by technology acceptance models, but the pandemic context involves also the use of a theory dealing with health risk. In addition, sharing personal glocalization may be considered as privacy concern leading to resistance rather than adoption. Even if the application is installed as requested, the user may not switch it on, which is a kind of hidden resistance.

A literature review led to the identification of an interesting but not widely verified framework, which has a great potential to explain the phenomenon under study: Social Amplification of Risk Framework (SARF). This theory is based on risk communication research and adopts a sociopsychological view regarding people reactions towards risky phenomena [2], [3], [4]. This framework can be extended and verified by focusing on the use of one new channel that reduces the contagion risk: COVID-19 Geo-localization mobile application.

The Risk amplification model of [2] can be used as an explanation of a social distancing behavior which is addressed through the adoption or resistance to COVID-19 geolocalization mobile applications. At the best of our knowledge, no article has applied this theory to explain the adoption or resistance towards preventive measures against COVID-19, whether they are classical (i.e., social distancing, wearing masks and etc.) or technological (i.e., adopting tracking mobile applications). This research aims to fill this gap by extending the social risk amplification model and adapting it to COVID-19 pandemic context. 


\section{Literature Review}

\subsection{Social Amplification of Risk Framework (SARF)}

This theory, which is an extension of research studies on communication risks, has a sociopsychological perspective regarding people's reactions to risks [2]. SARF explains how events or messages dealing with risk are augmented or diminished, knowing that the starting point is the information source and that communication channels may be individual, social or institutional [4], [5]. However, this framework has been criticized for its simplistic assumptions and for ignoring the way communication channels are used [2].

The Social risk amplification model posits that media exposure triggers risk perceptions and negative affect, which leads to information seeking and sharing. Based on these behaviors regarding information, people are engaged in preventive behaviors, to a certain extent. This model has been verified in a previous pandemic situation: the haze/dengue pandemic season in 2013. Thus, it may prove its validity in the COVID-19 context. This model is adapted and extended by including two types of reactions: social distancing prevented behavior, and resistance to a Covid related mobile application Tawakkalna and Tabaud.

The variables of the risk amplification model used in this research are: media exposure, perceived risk, affective reactions, information seeking and preventive behaviors or resistance.

\subsubsection{Media Exposure and Perceived Risk}

Traditional and social media impacts, as risk amplification factors, have been studied in the communication literature using qualitative studies [5] and quantitative ones [2]. [2] (p.1490) define traditional media as "popular, well-established media outlets" serving mainly channels which diffuse information. They include newspapers and radio in a comparative study including two issues related to health (Haze events vs Deng fever). They found that the impact of the score difference of traditional media exposure on the difference between risk perceptions is not supported in both cases. Unfortunately, they have not tested the impact of traditional media exposure on perceived risk in the case of Deng fever. The latter is a disease whose symptoms are like those of a common cold, but it can lead to death [2]. This prominent disease in SouthEast Asia affected about 2.5 billion people leading researchers to place it at the edge of an epidemic outbreak [6], [2]. This disease has therefore similarities with COVID-19, knowing that it has no known cure, and overcoming the disease relies mainly on the body immunity.

The impact of traditional media coverage on perceived risk has been verified in other diseases contexts like Bovine Spongiform Encephalopathy [5]. Accordingly, we expect that it will enhance the perception of risks in the context of COVID-19.

Compared to traditional media, electronic ones are interactive and connected among users [7]. Social Media (SM) which have a high level of interactivity can contribute to enhance the perceived risk pertaining to various social and health issues, and thereby serving as a platform leading to risk amplification via social pressure from social media users [2], [8], [9].

According to [2], SM networks could constitute a channel providing "social proof for performing certain behaviors" and "insights into normative reactions" (p.1491). Indeed, it may lead users to follow friends who shared information about preventative actions, or social media opinion leaders who have reacted a risky phenomenon. In the case of Dengue Fever epidemic, [2] verified that the exposure to social media has a greater effect on risk perceptions, compared to environmental issue phenomena like the haze event. Similarly, we expect that SM play an important role in enhancing the perception of risk related to the COVID-19 Pandemic. Accordingly:

H1- the media coverage about COVID-19 pandemic has a positive impact on the perception of risk.

\subsubsection{Affective Reactions}

Affective reactions are represented by emotions and moods which are stimulated by events and environments [10], [11]. They can be positive, when individuals feel enthusiasm, joy, pleasure or other types of positive feelings. They can be also negative when people feel sad, anxious, stressed... [12].

Affective reactions to pandemics have been considered by two theories: SARF [2], and the affective events theory [11].

[2] proposed a model based on SARF, in which negative affect are directly influenced by perceived risks which is, in turn influenced by media exposure. Although they have verified the difference between two situations (Dengue fever and Haze event), they affirmed that the correlations between these variables were significant. Using SARF theory, we can assume that negative affective reactions are triggered by perceived risk. Uncertainty is inherent to perceived risk and it is proposed as an appraisal base for affective reactions of individuals who face different risks [13], namely those related to pandemic-like problems [2]. 
The affective events theory assumes that affective reactions activated by event cognitions can be a significant force impacting people's behaviors and attitudes. According to this theory, affective reactions have a noteworthy mediating effect on the link between cognitive states and subsequent behavior [14]. Thus, they are stimulated by cognitions of an event [14], [11], which is appraised in this research by the perceived risk resulting from media exposure. Using this theory, we expect that those reactions are affected by the awareness of the dangerous situation based on social media exposure. Accordingly,

$\mathrm{H}$ 2: Negative affective reactions are influenced by perceived risk

\subsubsection{Behavioral Reactions in Corona Times}

In the context of a pandemic, the main objective of organizations and governments is to influence their population to adopt preventive behaviors like staying home, wearing masks, avoiding gatherings and crowds, and using geo-localization and social distancing applications. A theoretical support for the impact of affective reactions on preventive behaviors stems from the affective events theory for which affective reactions induced by important event, via cognitive appraisals, influence people's behaviors [14], [11].

In the context of Dengue fever pandemic, [2] used the concept of preventive behaviors as a consequence of negative affect. These behaviors include pushing family and friends to engage in preventive measures against the pandemic. This definition and assessment seem very general. We are using this general view and enriching it by including the use of new technologies as a way to avoid taking this virus. This includes using geo-localization and social distancing mobile applications.

Moreover, we consider information seeking about COVID-19 as a behavioral reaction which can be influenced by perceived risk and affective reactions induced by the awareness formed via media, whether they are social or traditional. Accordingly,

H3- Negative affective reactions triggered by COVID-19 pandemic have a positive impact on general preventive behaviors;

H4- Negative affective reactions triggered by COVID-19 pandemic have a positive impact on information seeking.

The adoption of the mobile applications dedicated to COVID-19, as a strategy to reduce the spread of the virus by using the geo-localization techniques, has been one of the strategies used in Saudi Arabia. Tawakkalna, launched in $2020 \mathrm{had}$ as a purpose to track Covid-19 infections. This application has not been fully used by the KSA population until government has made it mandatory. A decision has been made to allow entry to stores, malls, administrations, and only in condition of showing the home page of a mobile application entitled Tawakkalna. More than 200 million operations have been made on this mobile application within the 24 hours following the government's decision. The rush of users led to the lock out of already registered users. The app gradually coped with that problem and started working again after 24 hours [15]. This means that before this decision to make it mandatory, a big proportion of the public did not install it on their mobile phones and register in the Tawakkalna system. This might be explained by a lack of awareness about it or a certain resistance toward this application, as it is a way to provide a private information: the individual's location.

According to [16], the acceptance of innovative services by users is mainly affected by the success of strategies and solutions against resistance factors. We follow this idea by focusing on the resistance to mobile applications dedicated to COVID-19 rather than its adoption. Our study focuses on the period preceding the government decision to make the use of the application mandatory. A survey has been done just one month after the launch of the application.

[16] observe a lack in research studies focusing on consumer's resistance. They concluded that this is an explanation to the incomplete conceptualization of this construct.

Resistance to new technologies is considered as a normal response toward a change in habits if an innovation is adopted, especially when a great change is perceived. Indeed, people have a certain level of attachment to their prevailing behaviors, and most of them are unable to assume that a new habit or practice would be accepted [17], [18]. This leads to preventing the diffusion of the new technology and hampering the consumers' intention to use it [17]. Following [19], [17] argue that the resistance intensity influences the adoption postponement or the total product rejection. [18] add a third form of resistance to an innovation, namely opposition.

Resistance level is caused by the risk of an eventual change which is associated to the adoption of a new technology. This risk may be social, economic, and physical [16]. Indeed, users may identify a possible social harm associated with the possible localization of their mobiles and their persons, which may hinder their privacy. This should be compensated by the idea of minimizing others' physical risk related to COVID-19. Following the SARF model, the impact of risk is not direct, as it influences only affective reactions that have an impact on possible behaviors in the situation of a pandemic [2]. We advocate that the resistance vs. acceptance of geo-localization apps dedicated to 
COVID-19 pandemic are part of the behavioral reactions, which can enrich the SARF framework. It is likely to be affected by affective reactions.

H5- Negative affective reactions have an impact on the resistance against social distancing mobile applications.

The summary of the conceptual model is presented in Figure 1.

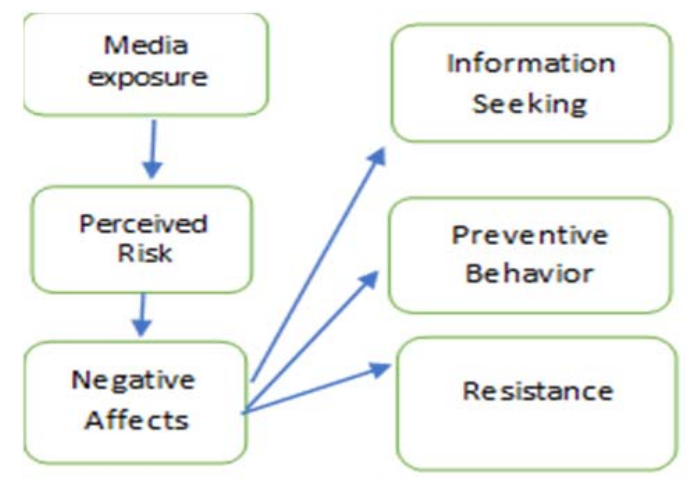

Figure 1. Conceptual Model

\section{Methodology}

This section highlights the research design and the measurement used.

\subsection{Research Design}

A survey using psychometric scales was distributed to Saudi citizens and expatriates via social media: WhatsApp, Instagram, and Facebook. This choice accelerated the data collection process, particularly when asking respondents to share the link with their network after answering the survey. Professionals, applying for a master during summer 2020, and faculty members in Saudi universities (Saudi Electronic University, King Saud University, Dar Al Uloom University and Al Imam University) have been asked to fill the survey and to share it with their families and social media groups. The targeted sample size was 400 to allow the use of structural equation modelling. This procedure has led to 312 valid observations: 118 for males and 194 for females. The sample structure is in the following Table (Table 1):

Table 1. Sample description

\begin{tabular}{|c|c|c|c|}
\hline Age & frequency & Profession & frequency \\
\hline below 18 & 19 & unemployed & 16 \\
\hline $18-29$ & 125 & $\begin{array}{c}\text { Employee } \\
\text { (private sector) }\end{array}$ & 75 \\
\hline $30-39$ & 92 & Students & 112 \\
\hline $40-49$ & 62 & $\begin{array}{c}\text { Employee } \\
\text { (government) }\end{array}$ & 99 \\
\hline $50-59$ & 10 & self-employed & 10 \\
\hline $65+$ & 1 & & \\
\hline Missing & 3 & & \\
\hline
\end{tabular}

\subsection{Measurements}

As shown in the conceptual model, the variables to be measured are: mass media and social media exposure, information seeking, preventive behavior, perceived risk and negative affective reactions, which are all measured via an adaptation of the measurement model of [2] who have already validated these measures in a similar context. A focus group involving two marketing experts and 6 volunteers selected from professionals applying for an MBA program have discussed the most important factors explaining the acceptance or resistance to measures against COVID-19 Pandemic, including the use of Tawakkalna. This also helped reducing the number of items corresponding to long measurement scales, such as perceived risk and resistance to the app use. In addition, an exploratory analysis led to the elimination of items with a low reliability.

The variable extending the SARF is the resistance to Tawakkalna. Its measure was adapted from [20]. After reduction, the final perceived risk, the preventive behavior, and the affective reaction measures [2] included three items each. Like media exposure, resistance included four items, while information seeking remained a bi-item scale. All items were evaluated on a 5 point-scale.

\section{Results}

\subsection{Measurement Model}

An exploratory factor analysis has first been made to check the dimensionality and reliability of measures. Then, we carried out a confirmatory factor analysis and checked the validity of measures.

The measurement model showed good fit indicators as the ratio between chi-square and the degree of freedom is low (2.06), knowing that Chisquare equals 282.44 with 137 degrees of freedom, as shown in Table 2. This Table also allows to verify the reliability and the validity, as it displays Average Variance Extracted (AVE), Standardized Loadings (SL), Alpha of Cronbach, and Composite Reliability (CoR). Indeed, table values indicate a satisfactory measurement model quality (all SL and CoR values above or equal to 0.7; all AVE values are greater than 0.5 ; all Alpha values are superior to 0.7 ). 
Table 2. Reliability and Validity indicators

\begin{tabular}{|c|c|c|c|c|c|}
\hline Items & Variables & SL & CoR & AVE & ALPHA \\
\hline \multirow{4}{*}{$\begin{array}{l}\text { Media } \\
\text { exposure }\end{array}$} & M2 & .744 & \multirow{4}{*}{0.858} & \multirow{4}{*}{0.603} & \multirow{4}{*}{0.875} \\
\hline & M3 & .818 & & & \\
\hline & M4 & .810 & & & \\
\hline & M5 & .729 & & & \\
\hline \multirow{4}{*}{ Resistance } & RESB2 & .823 & \multirow{4}{*}{0.857} & \multirow{4}{*}{0.601} & \multirow{4}{*}{0.874} \\
\hline & RESB4 & .698 & & & \\
\hline & RESB & .840 & & & \\
\hline & RESB3 & .730 & & & \\
\hline \multirow{3}{*}{$\begin{array}{l}\text { Preventive } \\
\text { Behavior }\end{array}$} & PCB3 & .818 & \multirow{3}{*}{0.901} & \multirow{3}{*}{0.752} & \multirow{3}{*}{0.90} \\
\hline & PCB2 & .916 & & & \\
\hline & PCB1 & .864 & & & \\
\hline \multirow{3}{*}{$\begin{array}{c}\text { Info } \\
\text { Seeking }\end{array}$} & InfS1 & .818 & \multirow{2}{*}{0.754} & \multirow{2}{*}{0.606} & \multirow{2}{*}{0.753} \\
\hline & InfS2 & .737 & & & \\
\hline & Aff1 & .856 & \multirow{3}{*}{0.896} & \multirow{3}{*}{0.743} & \multirow{3}{*}{0.927} \\
\hline \multirow[t]{2}{*}{$\begin{array}{l}\text { Affective } \\
\text { reactions }\end{array}$} & AFF2 & .878 & & & \\
\hline & AFF3 & .851 & & & \\
\hline \multirow[t]{3}{*}{ P. Risk } & RISK4 & .794 & \multirow{3}{*}{0.809} & \multirow{3}{*}{0.587} & \multirow{3}{*}{0.821} \\
\hline & RISK2 & .684 & & & \\
\hline & RISK5 & .814 & & & \\
\hline \multicolumn{6}{|c|}{$\begin{array}{c}\text { Model fit Indicators: GFI }=0.920, \text { NFI }=0.919 \text {, } \\
\text { IFI }=0.957, \text { RMSEA }=0.056\end{array}$} \\
\hline
\end{tabular}

Discriminant validity is verified in Table 3, which shows that values in the diagonal are greater than those in the correspondent row or column.

Table 3. Discriminant validity

\begin{tabular}{ccccccc}
\hline & RES & SME & RSK & affect & PRE & Inf.S \\
\hline RES & $\mathbf{0 . 7 7}$ & & & & & \\
\hline SME & 0.06 & $\mathbf{0 . 7}$ & & & & \\
\hline RSK & 0.30 & 0.28 & $\mathbf{0 . 7 6}$ & & & \\
\hline Affect & 0.23 & 0.22 & 0.50 & $\mathbf{0 . 8 6}$ & & \\
\hline PREV & 0.05 & 0.21 & 0.26 & 0.14 & $\mathbf{0 . 8 6}$ & \\
\hline Inf.S & 0.22 & 0.37 & 0.43 & 0.48 & 0.31 & $\mathbf{0 . 7 7}$ \\
\hline
\end{tabular}

\subsection{Structural Model Results and Discussion}

The final structural model has satisfactory fit indicators $\quad(\mathrm{GFI}=0.904 ; \quad \mathrm{IFI}=0.939 ; \quad$ Chisquare/ddl=2.38; RMSEA=0.064).

Table 4 shows the path estimates and indicates the significance of each path.

Table 4. Path estimates

\begin{tabular}{lccccc}
\hline Dependent & Independent & B & S.E. & P & Hyp \\
\hline Risk & $\begin{array}{c}\text { Media } \\
\text { Exposure }\end{array}$ & .402 & .087 & 0.00 & $\mathrm{H} 1$ \\
\hline $\begin{array}{l}\text { Affective } \\
\text { reactions }\end{array}$ & Risk & .585 & .071 & 0.00 & $\mathrm{H} 2$ \\
\hline $\begin{array}{l}\text { Info } \\
\text { seeking }\end{array}$ & $\begin{array}{c}\text { Affective } \\
\text { reactions }\end{array}$ & .483 & .068 & 0.00 & $\mathrm{H} 4$ \\
\hline $\begin{array}{l}\text { Preventive } \\
\text { behavior }\end{array}$ & $\begin{array}{l}\text { Affective } \\
\text { reactions }\end{array}$ & .142 & .051 & .006 & $\mathrm{H} 3$ \\
\hline $\begin{array}{l}\text { Resistance } \\
\text { to APP }\end{array}$ & $\begin{array}{l}\text { Affective } \\
\text { reactions }\end{array}$ & .161 & .072 & .026 & $\mathrm{H} 5$ \\
\hline
\end{tabular}

Results show that all hypotheses are accepted. Indeed, media exposure has a positive impact on perceived risk associated with COVID-19 ( $\beta=0.402$, $\mathrm{p}=0.00$ ), which leads us to accept $\mathrm{H} 1$.

This is in line with the approach of [2] based on social amplification theory. It also confirms the results of [5] who verified the link in another disease context. We can then confirm the importance of media exposure as a way to trigger greater levels of risk perception, which is a main factor leading to higher risk aversion and then a likely adoption of social distancing. In fact, perceived risk is also verified as a significant predictor of negative affective reactions $(\mathrm{p}=0.00)$, knowing that it has a positive impact on perceived risk $(\beta=0.585)$. Thus, $\mathrm{H} 2$ is accepted. This is also in line with the SARF and the results of [2]. Theoretically, it is explained by the fact that uncertainty is inherent to perceived risk and is an appraisal base for affective reactions of individuals who face different risks [13], [2]. This outcome also is in line with the theory of affective events, which assumes that affective reactions activated by event cognitions can be a significant force impacting people's attitudes. Indeed, affective reactions play an important role and are a result of cognitive states represented here by the perceived risk [14], [11].

The importance of affective states is also proved by the results related to $\mathrm{H} 3$, as negative affective reactions have a positive and significant impact on preventive behaviors against COVID-19 $(\beta=0.142$; $\mathrm{p}=0.006$ ). This is in accordance with two theories. The first is the theory of affective events showing the importance of affective reactions as mediators between events cognition and behavior, [14], [11]. The second is the SARF [2]. The verification of H5 and $\mathrm{H} 6$ are also in line with these theories as they involve two other forms of behavior significantly affected by affective reactions, namely the information seeking $(\beta=0.483, p=0.00)$ and the resistance to the use of mobile application associated with COVID-19 ( $\beta=0.161, p=0.026)$. However, the positive impact of negative affective reactions on resistance was not expected, as resistance to this app is theoretically a negative facet of preventive behaviors. People are supposed to adopt (contrary of resist) application that have been designed by government to limit the pace of the pandemic. Thus, negative affect, like fear from the consequences of the pandemic, is supposed to reduce the resistance to the tools acting for the same purpose. This means that other factors may intervene to explain this resistance, such as privacy concerns. 


\section{Conclusion}

This research aimed at verifying a model explaining the adoption of preventive behaviors against COVID-19 and the resistance to mobile geolocalization applications dedicated to this pandemic.

The main theoretical background of this model is the Social Amplification Risk Framework which has shown its reliability in other pandemic-like contexts, but not used in the context of COVID-19. This framework has been adapted and enriched by a new dependent phenomenon: the resistance to the use of mobile geo-localization apps like Tawakkalna which is dedicated to slow down the spread of the virus in Saudi Arabia.

Results have shown that social amplification framework is suitable for explaining such a phenomenon as all hypotheses have been accepted. The use of media has a crucial role in enhancing preventive behaviors, via the perceived risk and negative affective reactions. Thus, a managerial recommendation is to use media, advertisements and true stories that focus on the dark side of the pandemic. It highlights also the huge influence on individuals' and their loved ones' health.

Although negative affective reactions had a significant impact on the resistance to the chosen mobile application, its positive effect was surprising as we expected that fear from the pandemic would reduce the degree of resistance to the application. This leads us to think that other variables may be intervening to enhance this resistance, even in presence of pandemic anxiety. These variables may be the privacy and security concerns, the compatibility of the application to the mobile device, and the ease of use of that new technology. We have not integrated these variables which constitute a main limit of this research.

We propose to integrate technology acceptance or adoption models to the used framework to highlight the real predictors of the resistance to the mobile application. Variables like compatibility, ease of use, usefulness, habit, social influence would enhance the explanation power of the SARF model. These variables have a great potential to improve our model in future studies.

In addition, the use of media in our research was general and did not consider various types of communication strategies. We focused on the use of media, but not the content. It would be a value adding strategy to integrate different content strategies and their differential impacts: scenarios focusing on ones' risks vs. others' risks, scenarios focusing on fear vs. social responsibility, affective ads vs. or cognitive ads vs. news.

\section{Acknowledgements}

The authors extend their appreciation to the Deanship of Post Graduate and Scientific Research at Dar Al Uloom University for funding this work. (Fund based on the decision of the Council of the Deanship of Graduate Studies and Scientific Research at Dar Al Uloom University, taken in session 4on 13-12-2020. The time period of the project from 13 /12 / 2020 to 12/12/2021. Institution financing the project: Dar Al Uloom University/ Deanship of Post Graduate and Scientific Research.)

\section{References}

[1]. Bassi, A., Arfin, S., John, O., \& Jha, V. (2020). An overview of mobile applications (apps) to support the coronavirus disease 2019 response in India. Indian Journal of Medical Research, 151(5), 468. https://doi.org/10.4103/ijmr.ijmr 120020

[2]. Ng, Y. J., Yang, Z. J., \& Vishwanath, A. (2018). To fear or not to fear? Applying the social amplification of risk framework on two environmental health risks in Singapore. Journal of Risk Research, 21(12), 14871501.

[3]. Kasperson, R. E. (2012). The social amplification of risk and low-level radiation. Bulletin of the Atomic Scientists, 68(3), 59-66.

[4]. Kasperson, R. E., \& Kasperson, J. X. (1996). The social amplification and attenuation of risk. The annals of the American academy of political and social science, 545(1), 95-105.

[5]. Lewis, R. E., \& Tyshenko, M. G. (2009). The impact of social amplification and attenuation of risk and the public reaction to mad cow disease in Canada. Risk Analysis: An International Journal, 29(5), 714-728.

[6]. Gubler, D. J., \& Clark, G. G. (1995). Dengue/dengue hemorrhagic fever: the emergence of a global health problem. Emerging infectious diseases, 1(2), 55.

[7]. Sundar, S. S., Bellur, S., Oh, J., Jia, H., \& Kim, H. S. (2016). Theoretical importance of contingency in human-computer interaction: effects of message interactivity on user engagement. Communication Research, 43(5), 595-625.

[8]. Stieglitz, S., \& Dang-Xuan, L. (2013). Emotions and information diffusion in social media-sentiment of microblogs and sharing behavior. Journal of management information systems, 29(4), 217-248.

[9]. Gil de Zúñiga, H., Jung, N., \& Valenzuela, S. (2012). Social media use for news and individuals' social capital, civic engagement and political participation. Journal of computer-mediated communication, 17(3), 319-336.

[10]. Lazarus, R. S., \& Lazarus, R. S. (1991). Emotion and adaptation. Oxford University Press on Demand.

[11]. Mi, L., Zhao, J., Xu, T., Yang, H., Lv, T., Shang, K., ... \& Zhang, Z. (2021). How does COVID-19 emergency cognition influence public proenvironmental behavioral intentions? An affective event perspective. Resources, Conservation and Recycling, 168, 105467. https://doi.org/10.1016/j.resconrec.2021.105467 
[12]. Watson, D., Clark, L. A., \& Tellegen, A. (1988). Development and validation of brief measures of positive and negative affect: the PANAS scales. Journal of Personality and Social Psychology, 54(6), 1063-1070.

[13]. Frijda, N. H., Kuipers, P., \& Terschure, E. (1989). Relations among emotion, appraisal, and emotional action readiness. Journal of Personality and Social Psychology, 57, 212-228.

[14]. Weiss, H. M., \& Cropanzano, R. (1996). Affective events theory. Research in organizational behavior, 18(1), 1-74.

[15]. ARAB News, (2021). Retrieved from: https://www.arabnews.com/node/1804601/saudiarabia , [accessed: 14 March 2021].

[16]. Kim, J., Seo, J., Zo, H., \& Lee, H. (2020). Why digital goods have not replaced traditional goods: the case of e-books. Journal of Enterprise Information Management, 34(3), 793-810.
[17]. Laukkanen, P., Sinkkonen, S., \& Laukkanen, T. (2008). Consumer resistance to internet banking: postponers, opponents and rejectors. The International Journal of Bank Marketing, 26(6), 440455. https://doi.org/10.1108/02652320810902451

[18]. Szmigin, I., \& Foxall, G. (1998). Three forms of innovation resistance: the case of retail payment methods. Technovation, 18(6-7), 459-468.

[19]. Gatignon, H., \& Robertson, T. S. (1989). Technology diffusion: an empirical test of competitive effects. Journal of marketing, 53(1), 35-49.

[20]. Chouk, I., \& Mani, Z. (2019). Factors for and against resistance to smart services: role of consumer lifestyle and ecosystem related variables. Journal of Services Marketing, 33(4), 449-462.

https://doi.org/10.1108/JSM-01-2018-0046 